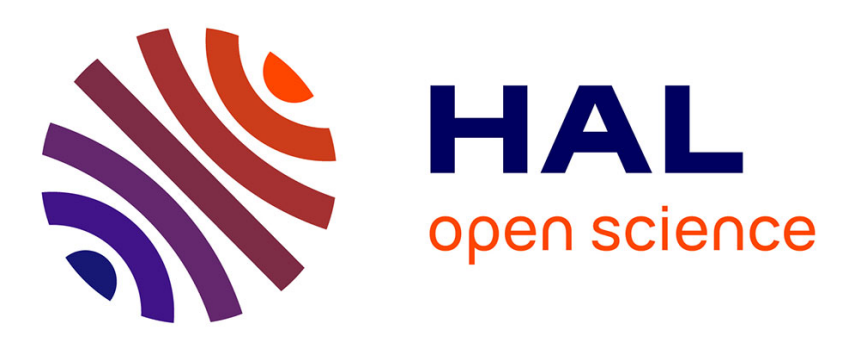

\title{
Two control laws for a spray system with time varying delay and dead band
}

\author{
Jan Anthonis, Wouter Franssens, Alexandre Seuret, Jean-Pierre Richard, \\ Herman Ramon
}

\section{> To cite this version:}

Jan Anthonis, Wouter Franssens, Alexandre Seuret, Jean-Pierre Richard, Herman Ramon. Two control laws for a spray system with time varying delay and dead band. IFAC World Congress 2005, Jul 2005, Prague, Czech Republic. pp.Th-A20-TO/4. hal-00385962

\section{HAL Id: hal-00385962 \\ https://hal.science/hal-00385962}

Submitted on 20 May 2009

HAL is a multi-disciplinary open access archive for the deposit and dissemination of scientific research documents, whether they are published or not. The documents may come from teaching and research institutions in France or abroad, or from public or private research centers.
L'archive ouverte pluridisciplinaire HAL, est destinée au dépôt et à la diffusion de documents scientifiques de niveau recherche, publiés ou non, émanant des établissements d'enseignement et de recherche français ou étrangers, des laboratoires publics ou privés. 


\title{
TWO CONTROL LAWS FOR A SPRAY SYSTEM WITH TIME VARYING DELAY AND DEAD BAND
}

\author{
Jan Anthonis ${ }^{* 1}$ Wouter Franssens * \\ Alexandre Seuret ${ }^{* *}$ Jean-Pierre Richard ${ }^{* *}$ \\ Herman Ramon* \\ * Laboratory of Agricultural Machinery and Processing, \\ K.U.Leuven. Kasteelpark Arenberg 30, 3001 Heverlee, \\ Belgium \\ ** LAGIS, Ecole Centrale de Lille, BP 48, 59651 \\ Villeneuve d'Ascq Cedex, France
}

\begin{abstract}
Two control laws, to control the liquid pressure of an agricultural spray boom are presented and compared. Characteristic for the process is a dead zone and a time varying delay. The first control law is rather intuitive and is based on direct dead zone compensation and a pole placement idea. The other control law originates from sliding mode control where the continuous part of the control contains some compensation dynamics, assuring stability. Both control laws are compared and tested in practice.
\end{abstract}

Keywords: Dead band, Time-varying delay, Sliding mode control, Stability, LMI

\section{INTRODUCTION}

In agriculture, spraying is one of the most important methods to distribute herbicides and liquid fertilizer to plants. Current practice is to distribute them as homogeneously as possible across the field to assure good biological efficiency. To compensate for irregularities in spray deposit due to speed variations, a control system adapts the flow rate to the speed. Recently, sensors to discriminate between weeds and plants have been developed (Vrindts et al., 2002), (Tian et al., 1999), (Wang et al., 2001) in research labs allowing sitespecific spraying i.e. spraying where the weeds are. This new method of spraying, puts new requirements to the spray hydraulics. Instead of spraying continuously, nozzles are opened and closed resulting into pressure variations. However, to assure

1 Post doctoral researcher from the fund of Scientific Research Flanders the right dose and correct droplet spectrum size, the pressure needs to be maintained as constant as possible. Very often, the dynamics of actual spray equipment may contain a variable time delay and a dead zone. The objective of this paper is to study control laws which can handle these phenomena.

Dead zones are often introduced by complex friction behaviour and deteriorate system performance. Several methods exist to handle friction in control systems. An overview is given in the work of Armstrong et al. (ArmstrongHélouvry et al., 1994) and Olsson et al.. (Olsson et al., 1998). They range from friction compensation based on accurate determined models, robust control methods like sliding mode e.g. (Hatipoglu and Özgüner, 1999), (Su et al., 2000), (Young et al., 1999) or adaptive algorithms, identifying online the friction e.g. (Ge and T.H. Lee, 2001), (Wang et al., 2004). 


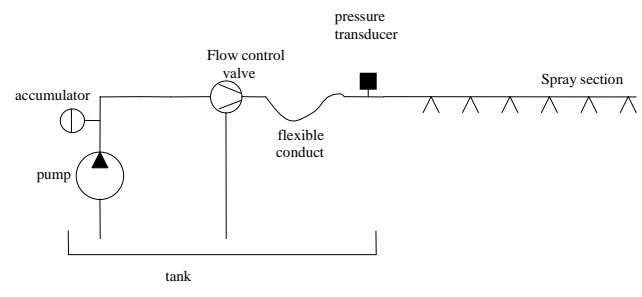

Fig. 1. Schematic representation of the pressure control system

About the study and control of systems with time delays, a good overview is given by Richard (Richard, 2003). Several methods exist to examine systems' stability with time varying delays. The Lyapunov-Razumikhin approach is known to be conservative (Cao et al., 1998) but can deal with time varying delays without any restriction on the derivative of the delay. With the LyapunovKrasovskii method, information about the time derivative used to be known until the work of Fridman et al. (Fridman et al., 2004) and Richard et al. (Richard et al., 2001). As their results are promising, this approach is followed in the paper.

The organisation of the paper is as follows. First, a description of the system is provided and a model is presented. Two control laws are proposed and compared. Stability issues are discussed and finally, practical implementation results are shown.

\section{SYSTEM DESCRIPTION}

Figure (1) shows the lay out of the system. It is actually one section of an agricultural spray boom for application of herbicides and fertilizer to the plants. A pump, containing two pistons, operating in anti-phase, feeds the circuit. Pressure peaks, resulting from fast activation of valves or originating from the pulsating flow of the pump, are attenuated by the accumulator. The pressure at the nozzles is regulated by a flow control valve by adjusting the opening to the return. A long flexible conduct links the pressure control valve with the metal conduct, on which the nozzles are mounted. An electronic transducer measures the pressure at the entrance of the metal conduct. This is the pressure of interest which is measured and should be controlled. The system is secured by a check valve, limiting the pressure to 7bar.

In the laboratory, the total non-linear system has been modelled and validated. For controller design, the following reduced model, which represents actually the slow dynamics, in the sense of singular perturbed systems (Khalil, 2002), is sufficient.

$$
\begin{gathered}
\dot{x}=K_{m} f_{d}(u) \\
\sqrt{y}=\frac{\alpha x(t-h)+\beta}{x(t-h)+\gamma} 0 \leq h_{\min } \leq h \leq h_{\max }
\end{gathered}
$$

The only relevant state $x$ is the angle of the valve, which is actuated by an electrical DC motor. A complex mechanism which originates from compliance of the motor struts, which is needed for assembly reasons, introduces the time varying delay. Therefore, the time delay changes by the change of direction of the electrical motor. Due to dry friction between the ball of the valve and the housing a dead zone $f_{d}(u)$ occurs.

$$
\begin{array}{ccc}
f_{d}(u)=u-c_{0} \operatorname{sign}(u) & \text { if } & c_{1} \leq|u|, \\
f_{d}(u)=0 & \text { if } & c_{1}>|u|, \\
\text { and } & 0 \leq c_{0} \leq c_{1} .
\end{array}
$$

The dead zone model $f_{d}(u)$ is clearly a simplification of the underlying friction behaviour.

\section{STATE ESTIMATOR DESIGN}

In order to stabilize the state equation in (1), the rotation angle $x$ of the valve should be known. The valve doesn't contain a measurement system to determine the rotation angle, such that only the delayed state $x(t-h)$ is available from the output $\sqrt{y}$. Applying directly the delayed state $x(t-h)$ in a control law leads to limit cycle behaviour. Therefore, a state estimator is constructed.

$$
\dot{\hat{x}}(t)=K_{m} f_{d}(u)+E(x(t-h)-\hat{x}(t-\hat{h}))
$$

where $\hat{x}$ is an estimate of the state $x, \hat{h}$ an estimate of the delay $h$ and $E$ the Kalman gain. Actually, equation (3) can only have an interpretation of a Kalman filter if $h=\hat{h}$.

\section{FIRST CONTROL LAW}

Based on the prediction of the state $\hat{x}$, the control law is constructed:

$$
u=-\frac{1}{K_{m}}\left(u_{d} \operatorname{sign}\left(\hat{x}(t)-\hat{x}_{d}\right)+a\left(\hat{x}(t)-\hat{x}_{d}\right)\right)
$$

Constant $\hat{x}_{d}=\frac{\beta-\gamma \sqrt{p_{d}}}{\sqrt{p}_{d}-\alpha}$ is the desired rotation angle, calculated back through the second part of equation (1) by replacing $y$ by the desired pressure $p_{d}$. Constants $a$ and $u_{d}$ are design parameters. 
Such a simple control law, with direct dead band compensation, is often found back in practical control systems. It is based on the idea of pole placement, in which $a$ is the final pole location, when the dead zone is compensated perfectly and $c_{1}=c_{0}$ equal $u_{d} / K_{m}$. However, in order to ensure that the dead zone is compensated, $u_{d} / K_{m}$ should be larger than $c_{1}$. Therefore the determination of the parameters of the observer and control law should be based on the theory of sliding modes. From equation (4) the sliding surface $s(t)$ can be derived:

$$
s(t)=\hat{x}(t)-\hat{x}_{d}
$$

In sliding regime, the observer (3) is in sliding mode and the dynamics of the reduced system is:

$$
\dot{x}=-E(x(t-h)-\hat{x}(t-\hat{h}))
$$

It is striking that during sliding, the dynamics of the system are governed by gain $E$ of the predictor and not by gain $a$ of the control law in equation (4). By the simple reasoning of perfect compensation and pole placement, $a$ was faulty thought to determine the closed loop pole. In order to ensure a stable sliding mode, constant $E$ should be determined such that equation (6) is stable. The state in equation (6) contains a time delay $h$, which changes by altering the direction of $x(t)$. In the worst case, $h$ may change discontinuously such that proper selection of gain $E$ is not straightforward. Next theorem provides a sufficient condition on $E$ such that equation (6) is stable.

Theorem 1. Let $E \geq 0$, if :

$$
\frac{\pi}{2 h_{\max }}>E,
$$

the system in equation (6) is stable with bounded delay: $h \leq h_{\max }$

Proof:

See (Anthonis et al., n.d.)

To formulate the stability condition on the design parameters of the observer and control law, two lemma's are required.

Lemma 1. Let the delay of the system and observer satisfying $h, \widehat{h} \leq h_{\max }$ and the size of the delay $h$ only changes by the change of the direction of the angle $x$ of the control valve. The sliding line $s(t)$ given by equation (5) is attractive and reached in finite time for the control law in equation (4). However, once the system is on the sliding line, it is not ensured that it remains on it.

Proof:

See (Anthonis et al., n.d.)

Lemma 2. The system given by

$$
\dot{q}=A_{0} q(t)+A_{1} q(t-h)+A_{2} q(t-\hat{h})
$$

with

$$
\begin{gathered}
A_{0}=\left(\begin{array}{cc}
0 & 0 \\
0 & -a
\end{array}\right) A_{1}=\left(\begin{array}{cc}
-E & -E \\
E & E
\end{array}\right) \\
A_{2}=\left(\begin{array}{cc}
0 & E \\
0 & -E
\end{array}\right)
\end{gathered}
$$

with time varying delays $h, \hat{h}$ and $h \leq h_{\max }$ $\hat{h} \leq \hat{h}_{\max }$ is stable if there exist $2 \times 2$ matrices $0<P_{1}, P_{2}, P_{3}, Z_{1 i}, Z_{2 i}, Z_{3 i}$ and $R_{i}, i=1,2$ that satisfy the following LMIs:

$$
\Psi_{1}<0 \text { and }\left(\begin{array}{cc}
R_{i} & \left(\begin{array}{cc}
0 & A_{i}
\end{array}\right) P \\
* & Z_{i}
\end{array}\right) \geq 0
$$

where

$$
\begin{gathered}
P=\left(\begin{array}{cc}
P_{1} & 0 \\
P_{2} & P_{3}
\end{array}\right) Z_{i}=\left(\begin{array}{cc}
Z_{1 i} & Z_{2 i} \\
* & Z_{3 i}
\end{array}\right) \\
\Psi_{1}=\Psi_{0}+\sum_{i=1,2}\left(\begin{array}{cc}
0 & 0 \\
0 & h_{\max } R_{i}
\end{array}\right)+h_{i} Z_{i} \\
h_{1}=h_{\max } h_{2}=\hat{h}_{\max } \\
\Psi_{0}=P^{T}\left(\begin{array}{cc}
0 & I \\
A & -I
\end{array}\right)+\left(\begin{array}{cc}
0 & I \\
A & -I
\end{array}\right)^{T} P
\end{gathered}
$$

and

$$
A=A_{0}+A_{1}+A_{2}
$$

Proof:

The theorem is an extension of a theorem of Fridman (Fridman et al., 2004).

Theorem 2. The control law (4) makes the surface $s(x)=0$ attractive and reached in finite time. The equilibrium $x=\hat{x}_{d}$ is then globally asymptotically stable for $h \leq h_{\max } \hat{h} \leq \hat{h}_{\max }$ provided that $E<\frac{\pi}{2 h_{\max }}$ and if there exist $2 \times 2$ matrices $0<P_{1}$, $P_{2}, P_{3}, Z_{1 i}, Z_{2 i}, Z_{3 i}$ and $R_{i}, i=1,2$ that satisfy the LMIs (9) and $P_{1}$ diagonal. 
Proof:

First the system equations (1), (3) are transformed into regular form:

$$
\begin{gathered}
\dot{e}(t)=-E e(t-h)-E s(t-h)+E s(t-\hat{h}) \\
\dot{s}(t)=E e(t-h)+E s(t-h)-E s(t-\hat{h})+ \\
K_{m} f_{d}(u)
\end{gathered}
$$

With the control law (4) this results in:

$$
\begin{gathered}
\dot{q}=A_{0} q(t)+A_{1} q(t-h)+A_{2} q(t-\hat{h}) \\
-\left(u_{d}-K_{m} c_{0}\right) \operatorname{sign}\left(\left(\begin{array}{lll}
0 & 1
\end{array}\right) q(t)\right)
\end{gathered}
$$

Similar to the proof of lemma 2 and (Fridman et al., 2004), the system is transformed to descriptor form and the same Lyapunov-Krasovskii functional is selected to proof stability. After elaboration, this results in:

$$
\dot{V}(t)=\dot{V}^{*}(t)-2 P_{11}\left(u_{d}-K_{m} c_{0}\right)|s(t)|
$$

in which $\dot{V}^{*}(t)$ is the derivative of $V(t)$ in case equation (11) doesn't contain the sign term on the right hand side i.e., the system of equation (8). $P_{11}$ is the first row, first column element of $P_{1}$. Provided the LMIs $(9)$ are satisfied, $\dot{V}^{*}(t)<$ 0 therefore $\dot{V}(t)<0$. Moreover, the system is globally asymptotically stable, as $V^{*}\left(q_{e}, y_{e}\right)=0$ (Fridman et al., 2004), $V\left(q_{e}, y_{e}\right)=0$, with $q_{e}$ and $y_{e}$ the equilibrium states and $V(q, y) \rightarrow \infty$ for $\left\|\left(q^{T} y^{T}\right)\right\| \rightarrow \infty$. Furthermore, the switching line is reached in finite time by the stability of the exponential stability of the system and theorem 1.

On the switching line $s(x)=\dot{s}(x)=0$ such that, given the regular form equations (10) the reduced system is governed by the following equation:

$$
\dot{e}(t)=-E e(t-h)
$$

which has been proved stable with the requirement (7) by theorem 1 .

\section{SECOND CONTROL LAW}

The first control law (4) was rather intuitive. The second control law relies on the same switching line (5) but is synthesised, based on design methods, used in the theory of the sliding modes which often employ the regular form as in equation (10).

The dead zone $f_{d}(u)$ can be considered as the input $u(t)$ and a disturbance $d(t)$ which leads to:

$$
\dot{s}(t)=E e(t-h)+K_{m} u(t)+K_{m} d(t)
$$

From equation (2), it is easy to see that $d(t)$ is bounded:

$$
|d(t)|<c_{1}
$$

The control law is inspired on the work of Richard (Richard et al., 2001), who proposed variable structure control laws for linear time invariant systems with time varying delay and bounded disturbance.

$$
\begin{gathered}
u(t)=-\frac{1}{K_{m}}\{E e(t-h)+E s(t-h)-E s(t-\hat{h})+ \\
\left.u_{d} \operatorname{sign}(s(t))+a s(t)\right\}
\end{gathered}
$$

where constants $u_{d}$ and $a$ are defined as

$$
u_{d}=m_{1}+K_{m} c_{1}
$$

and constants

$$
a, m_{1}>0
$$

Control law (16) is equivalent to:

$$
\begin{gathered}
u(t)=-\frac{1}{K_{m}}\left\{E(x(t-h)-\hat{x}(t-\hat{h}))+u_{d} \times\right. \\
\operatorname{sign}(s(t))+a s(t)\}
\end{gathered}
$$

This control law is apart from the term $E(x(t-$ $h)-\hat{x}(t-\hat{h}))$ exactly the same as the first control law (4) and can easily be implemented as $x(t-h)$ is, through $\sqrt{y}$, readily available from the measurement. In the first control law (4), the discontinuous term is selected such that $|u(t)| \geq$ $c_{1}$, in order to assure that whenever a control action is desired, it really reaches the system and is not blocked by the dead zone. Note that this requirement is not necessarily fulfilled in the second control law (16). Nevertheless, asymptotic stability is achieved.

Theorem 3. A) The control law (16) makes the surface (5) attractive and reached in finite time for the state equations (1), (3).

B) The equilibrium $x_{1}=\hat{x}_{1}=\hat{x}_{d}$ is then globally asymptotically stable for $h_{\min } \leq h \leq h_{\max }$, bounded delay estimation $\hat{h}$ and for $E$ satisfying inequality (7).

Proof: 
For part A), the following Lyapunov function is selected:

$$
V(t)=s(t) s(t)
$$

Its derivative along the solution of (1), (3) is:

$$
\begin{gathered}
\dot{V}(t)=s(t)(E e(t-h)+E s(t-h)- \\
\left.E s(t-\hat{h})+K_{m} u(t)+K_{m} d(t)\right)
\end{gathered}
$$

Inserting control law (15) renders:

$$
\dot{V}<-2 m_{1} \sqrt{V}-2 a V<-2 m_{1} \sqrt{V}
$$

which proves that $s(t)=0$ is a sliding surface, reached in finite time.

The proof of part $\mathrm{B}$ of the theorem relies on the attraction of the sliding line and by the fact that equation (22) is always valid such that once $s(t)=\dot{s}(t)=0$, by the bounded delays $h$ and $\hat{h}$, also $s(t-h)$ and $s(t-\hat{h})$ evolve in finite time to zero. Therefore in sliding mode, the reduced system corresponds to equation (13), of which the stability has already been proved by theorem 1 .

The stability conditions of the second control law (16) are less restrictive than those of the first control law (4). However for both laws, the conditions are sufficient and it is not clear how conservative they are.

\section{IMPLEMENTATION}

The observer and the two controllers are programmed on a digital controller (ADWIN Gold, Jaeger GMBh). As a discretisation rule, the zero order hold transformation was utilized. In order to approximate the behaviour of a continuous controller, a rather high sampling frequency of $1000 \mathrm{~Hz}$ has been selected. It ensures also that the Shannon principle is not violated. The two piston membrane pump generates harmonics of $23 \mathrm{~Hz}$.

Table 1. System parameters and parameters of the control law.

\begin{tabular}{lll}
\hline$K_{m}\left({ }^{\circ} /(\mathrm{Vs})\right)$ & $\alpha\left(\sqrt{P a} /{ }^{\circ}\right)$ & $\beta(\sqrt{P a})$ \\
1.71 & $1.59 \times 10^{3}$ & $-1.04 \times 10^{5}$ \\
\hline$\gamma\left(^{\circ}\right)$ & $c_{0}(V)$ & $u_{d}(V)$ \\
\hline-53.9 & 2.72 & 4 \\
\hline
\end{tabular}

All relevant parameters of the system are listed in table 1. As stated in the introduction, the delay changes by changing the direction of rotation of the valve. However, there is no fixed value of the delay for a certain direction. After some experiments, a maximum delay of $0.2 s$ and a minimum delay of $0.1 s$ has been observed. The estimation of the time delay $\hat{h}$ is selected fixed and equal to the average of the maximum and minimum delay: $0.15 s$. Parameter $a=5.85$ is determined as high as possible, avoiding input saturation. Applying theorem 1 results in a maximum $E$, assuring stability, of 7.85 . With respect to stability of the first control law, the LMIs of theorem 2 need also to be verified allowing a maximum $E$ of about 5.6. By decreasing $\hat{h}$, the maximum value of $E$ increases. In case $\hat{h}$ is put equal to zero, $E$ can be enlarged up to 7 . However, experiments on the real set-up show a better performance and a more stable behaviour with $\hat{h}=0.15$ instead of $\hat{h}=0$. With $\hat{h}=0.15$, gain $E$ could be increased more before obtaining unstable behaviour. Simulations in Matlab confirm this. The simulations are based on the system model (1) and a delay $h$ which can only take two distinct values $(0.1 s$ and $0.2 s)$. This seeming contradiction between theory and practice can be explained by the fact that theorem 2 just considers the maximum delay values of $h$ and $\hat{h}$. In practice, there is an important relation between these two i.e. the one is the prediction of the other. Therefore the class of systems considered by theorem 2 is much larger than required such that conservative results are obtained. For the second controller (16), decreasing the delay estimate $\hat{h}$ to zero gives an increase in performance, without affecting stability. A possible explanation for this is that the stability of this control law is independent of the size of $\hat{h}$. Only a bounded $\hat{h}$ is required. The delay estimation $\hat{h}$ affects the term $s(t-\hat{h})$ in the control law, reflecting the distance to the sliding line in the past. Therefore, it is clear that for performance $\hat{h}$ is the best as small as possible.

The two control laws are compared in figures 2 and 3 for $E=7$ and $\hat{h}=0.15$ respectively $\hat{h}=0$. The oscillations on the figure, are due to the piston pump. Based on the figures, both controllers show the same performance. In practice, the first controller (4) seems to react more accurate than the second (16). For some set-point pressures, it shows some off-set and on small set-point jumps $(<0.2 b a r)$ it doesn't react.

To investigate stability, gain $E$ is increased until unstable behaviour is obtained. In case of the first controller (4), taking $E$ larger than 8 showed sometimes strange behaviour and after 11, the pressure went to its maximum value. For the second controller (16), even after increasing $E$ to 30 , apart from decaying oscillatory behaviour, no instability was observed. This can only be explained by the fact that the error $e(t-h)$ needs to be zero before reaching the switching line. 


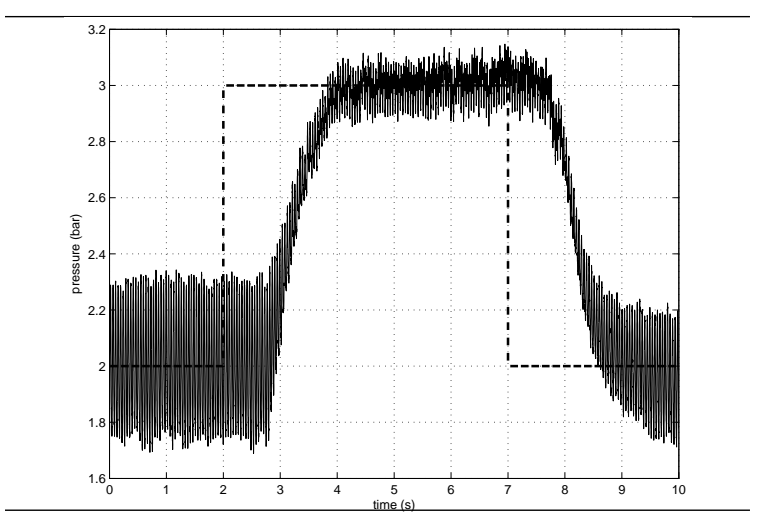

Fig. 2. Measured (full line) and desired (dashed line) pressure for the first controller (4)

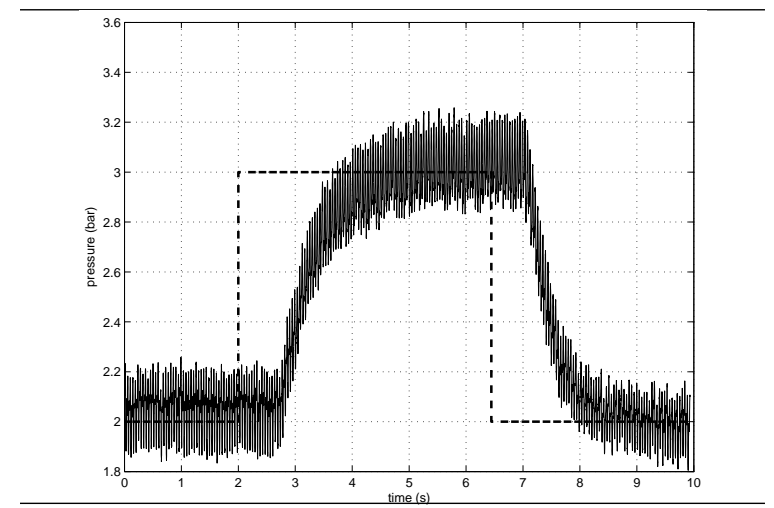

Fig. 3. Measured (full line) and desired (dashed line) pressure for the first controller (16)

\section{CONCLUSIONS}

Two control laws, that can cope with dead zones and time varying delays, to stabilize the pressure in a hydraulic circuit for spraying pesticides have been proposed. The first control law ensures that the control signal is always larger than the dead zone. It is intuitive but following this intuition may lead to bad design. Despite its intuitive character, it is more difficult to prove the stability. The second control law doesn't guarantee a control output larger than the dead zone but by its construction, asymptotic stability is acquired. In practice, the first control law shows a slightly better performance but the second control law is more stable. With respect to the delay estimation, requires the first control law a good estimate to guarantee a good performance and stable behaviour. In case of the second control law, the delay estimate should be just considered as a parameter and put to zero.

\section{REFERENCES}

Anthonis, J., A. Seuret, J.P. Richard and H. Ramon (n.d.). Réglage d'un processus de pulvérisation caracterisé par une zone morte et un retard.

Armstrong-Hélouvry,

B.,

P. Dupont and C. Canudas de Wit (1994). A survey of models, analysis tools and compensation methods for the control of machines with friction. Automatica 30(7), 1083-1138.

Cao, Y., Y. Sun and C. Cheng (1998). Delaydependent robust stabilization of uncertain systems with multiple state delays. IEEE Transactions on Automatic Control 43(11), 1608-1612.

Fridman, E., A. Seuret and J.P. Richard (2004). Robust sampled-data stabilization of linear systems: An input delay approach. Automatica 40(8), 1441-1446.

Ge, S.S. and S.X. Ren T.H. Lee (2001). Adaptive friction compensation of servo mechanisms. International Journal of Systems Science 32(4), 523-532.

Hatipoglu, C. and Ü. Özgüner (1999). Handling stiction with variable structure control. In: Lecture notes in control and information science 247, Variable structure systems, Sliding Mode and Nonlinear Control. pp. 143-166. Springer-Verlag. London.

Khalil, H.K. (2002). Non-linear Systems. Pearson Higher Education. Prentice Hall.

Olsson, H., K.J. Astrőm, C. Canudas de Wit and P. Lichinsky M. Gäfvert (1998). Friction models and friction compensation. European Journal of Control (4), 176-195.

Richard, J.P. (2003). Time-delay systems: an overview of some recent advances and open problems. Automatica 39(10), 1667-1694.

Richard, J.P., F. Gouaisbout and W. Perruquetti (2001). Sliding mode control in the presence of delay. Kybernetika 37(3), 277-294.

Su, A.-C., S.V. Drakunov and Ü. Özgüner (2000). An o(t2) boundary layer in sliding mode for sampled-data systems. IEEE Transactions on Automatic Control 43(3), 482-485.

Tian, L., J.F. Reid and J.W. Hummel (1999). Development of a precision sprayer for site-specific weed management. TRANSACTIONS OF THE ASAE 42(4), 893-900.

Vrindts, E., J. De Baerdemaeker and H. Ramon (2002). Weed detection using canopy reflection. Precision Agriculture 3(1), 63-80.

Wang, N., N. Zhang, F.E. Dowell, Y. Sun and D.E. Peterson (2001). Design of an optical weed sensor usingplant spectral characteristics. TRANSACTIONS OF THE ASAE 44(2), 409-419.

Wang, X.-S., C.-Y. Su and H. Hong (2004). Robust adaptive control of a class of nonlinear systems with unknown dead-zone. Automatica 40(3), 407-413.

Young, D.K., V.I. Utkin and Ü. Özgüner (1999). A control engineer's guide to sliding mode control. IEEE Transactions on Control Systems Technology 7(3), 328-342. 